\title{
Arrhythmia susceptibility in senescent rat hearts
}

\author{
Stefano Rossi, ${ }^{1}$ Silvana Baruffi, ${ }^{1}$ Domenico Corradi, ${ }^{2,3}$ Sergio Callegari, ${ }^{4}$ Ezio Musso, ${ }^{1}$ \\ Andrea Sgoifo, ${ }^{5}$ Luca Carnevali, ${ }^{5}$ Michele Miragoli, ${ }^{3}$ Emilio Macchi ${ }^{1,3}$ \\ ${ }^{1}$ Department of Biosciences, University of Parma; ${ }^{2}$ Department of Biomedical, Biotechnological \\ and Translational Sciences, University of Parma; ${ }^{3}$ Center of Excellence of Toxicological Research, \\ University of Parma; ${ }^{4}$ Department of Cardiology, Vaio Hospital, Fidenza; ${ }^{5}$ Department of \\ Neurosciences, University of Parma, Italy
}

\begin{abstract}
Cardiovascular disease increases with age as well as alterations of cardiac electrophysiological properties, but a detailed knowledge about changes in cardiac electrophysiology relevant to arrhythmogenesis in the elderly is relatively lacking. The aim of this study was to determine specific age-related changes in electrophysiological properties of the ventricles which can be related to a structural-functional arrhythmogenic substrate. Multiple epicardial electrograms were recorded on the ventricular surface of in vivo control and aged rats, while arrhythmia vulnerability was investigated by premature stimulation protocols. Single or multiple ectopic beats and sustained ventricular arrhythmias were frequently induced in aged but not in control hearts. Abnormal ventricular activation patterns during sinus rhythm and unchanged conduction velocity during point stimulation in aged hearts suggest the occurrence of impaired impulse conduction through the distal Purkinje system that might create a potential reentry substrate.
\end{abstract}

Correspondence: Stefano Rossi, Department of Biosciences, University of Parma, Parco Area delle Scienze 11A, 43124 Parma, Italy.

Tel. +39.0521.902111 - Fax: +39.0521 .347038 .

E-mail: stefano.rossi@unipr.it

Key words: aged heart, arrhythmia, epicardial mapping.

Acknowledgements: we acknowledge Riccardo Labadini, Department of Biosciences, University of Parma, Italy, for his excellent technical assistance. This research was supported by Fondazione CARIPARMA Grant 2006.0082, Grant September 2010 and Grant GR2009-1520528 Young Researcher Grant, Italian Ministry of Health to MM.

@C Copyright S. Rossi et al., 2014

Licensee PAGEPress, Italy

Journal of Biological Research 2014; 87:2143

doi:10.4081/jbr.2014.2143

This article is distributed under the terms of the Creative Commons Attribution Noncommercial License (by-nc 3.0) which permits any noncommercial use, distribution, and reproduction in any medium, provided the original author(s) and source are credited.

\section{Introduction}

Cardiovascular disease increases with age. Significant alterations of cardiac electrophysiological properties occur during the normal aging process as a result of structural remodeling of the extracellular matrix, modifications of cell-to-cell connection between neighboring cardiac myocytes (gap junctions) and changes in active membrane properties. Even if advanced age appears to be a risk factor for increased susceptibility to ventricular arrhythmia, a detailed knowledge about changes in cardiac electrophysiology relevant to arrhythmogenesis in the elderly is relatively lacking.

Thus, the aim of the study was to determine specific age-related changes in electrophysiological properties and myocardial structure of the ventricles which can be related to a structural-functional arrhythmogenic substrate.

\section{Materials and Methods}

Multiple epicardial unipolar electrograms were recorded on the ventricular surface of in vivo control (10 months) and aged (24 months) rats during normal sinus rhythm and ventricular pacing by means of a 8x8 electrode array ( $1 \mathrm{~mm}$ resolution) obtained by fastening insulated silver wire on a surgical cotton gauze. The electrode array was positioned on the anterior ventricular surface in open chest rats. Arrhythmia inducibility was tested by single or multiple premature stimulation and burst pacing in uniformly spaced array electrodes. Fiber architecture, connexin (Cx) 43, Cx40, interstitial fibrosis and tissue collagen content were determined by (immune)histology, while proteins content was performed by Western blotting.

\section{Results}

Spontaneous supraventricular and ventricular rhythm disturbances were frequently observed in aged hearts ${ }^{1}$ during normal sinus rhythm. Ectopic beats during premature stimulations and sustained ventricular arrhythmias during burst pacing were frequently induced in aged hearts but not in control hearts (Figure 1).

Electrophysiological analysis in aged hearts revealed that PQ duration and total ventricular activation time (QRS duration) were significantly delayed as compared to control hearts while anisotropic conduction velocity did not change significantly. ${ }^{2}$ Ventricular activation pat- 


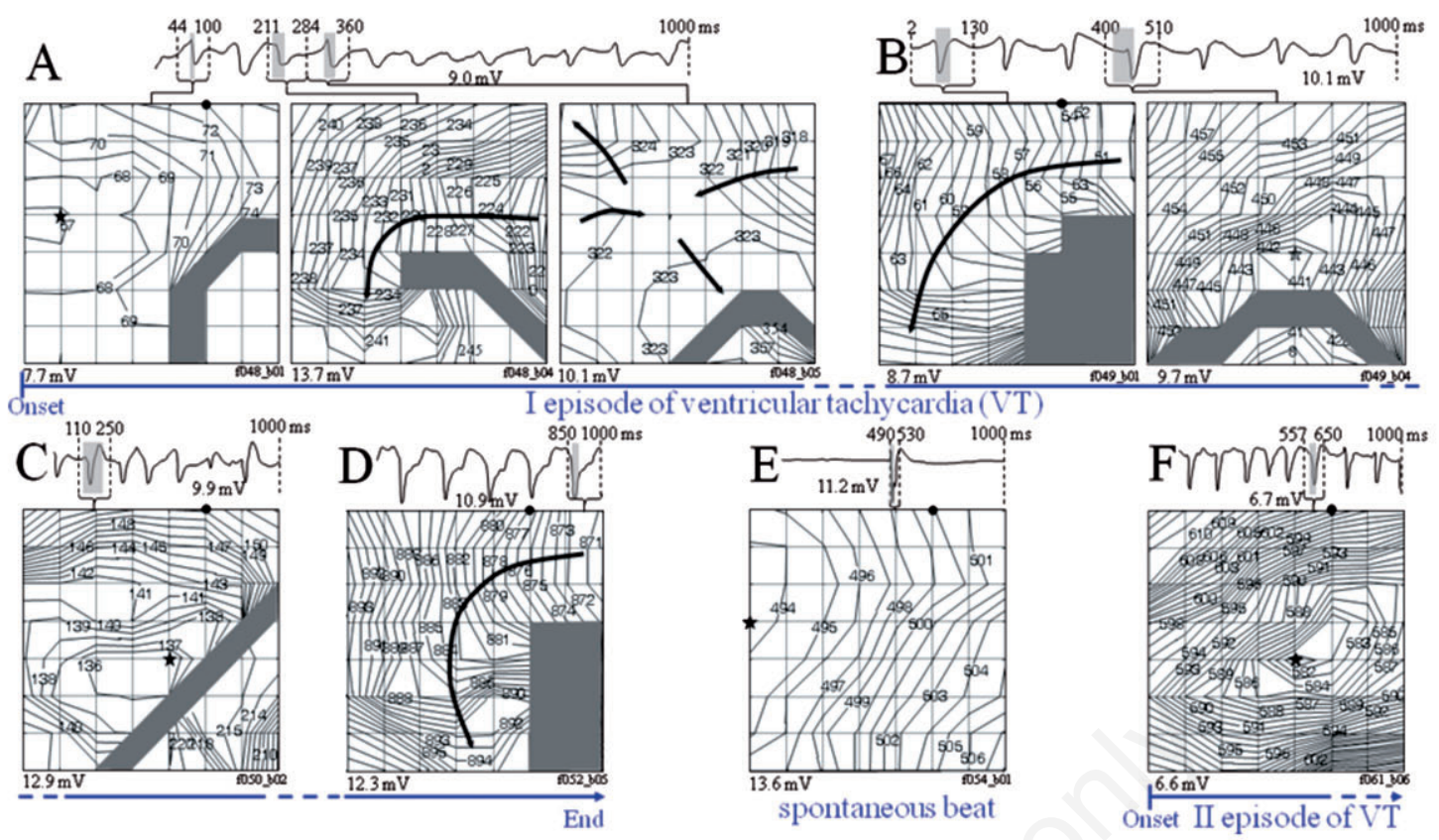

Figure 1. A) Different cycles of arrhythmia events in an aged heart characterized either by slow propagating wavefronts circulating around a functionally refractory region or by multiple wavefronts. B) Subsequent cycles of the arrhythmia are characterized by slow circus movement of reentrant activation sequences around the same region of functional block or by focal activation spreading with slow anisotropic conduction velocity. C) Subsequently, some of the cycles display the same focal origin as in previous arrhythmic episodes and interestingly the focal activation is localized in the same point of the breakthrough point during sinus rhythm. D) The arrhythmia terminates with a $5 \mathrm{~Hz}$ monomorphic sequence characterized by wavefronts circulating counter-clockwise around an obstacle in the lower right area of the electrode array. E) Arrhythmia terminates with spontaneous ventricular activation characterized by low heart rate. F) During a II episode of ventricular tachycardia few beats of focal activation spreading with slow anisotropic conduction velocity are observed. In each panel, above: reference EG; below: isochrone maps. In each reference EG, $1 \mathrm{~s}$ duration, numbers in ms=time from beginning of EG; number in $\mathrm{mV}=$ maximum peak-to-peak voltage; pair of braced vertical dotted lines=confidence time interval for minimum derivative computation; curly brace and segmental line=link between reference EG activation time interval (shaded area) and corresponding isochrone map. In each isochrone map, numbers in $\mathrm{ms=time}$ from reference EG beginning; black star=breakthrough point; black dot=reference EG electrode; grey area=functional conduction block; arrows=wavefront directions; VT=ventricular tachycardia.

terns during sinus rhythm displayed variable number, location and time of appearance of epicardial breakthrough points. ${ }^{2}$

Morphological analysis in aged hearts revealed that heart weight and myocyte transverse diameter increased significantly, scattered microfoci of interstitial fibrosis were mostly present in the ventricular subendocardium and gap junction $\mathrm{Cx} 43$ and $\mathrm{Cx} 40$ expression decreased significantly in ventricular myocardium, as compared to controls. ${ }^{2}$

\section{Discussion}

Delayed QRS duration, abnormal activation patterns and unchanged ventricular conduction velocity in aged hearts suggest the occurrence of impaired impulse conduction through the Purkinje system leading to uncoordinated myocardial excitation despite structural changes. Impaired interaction between Purkinje system and ventricular myocardium might create a potential reentry substrate, contributing to higher vulnerability to ventricular arrhythmias observed in aged hearts. This is consistent with the occurrence of intermittent focal anisotropic activations, possibly originating from Purkinje-ventricular junctions, among sequences of wide wavefront circus movements around a functionally refractory region.

\section{References}

1. Rossi S, Baruffi S, Bertuzzi A, et al. Susceptibility to ventricular arrhythmias in aged hearts. Conf Proc IEEE Eng Med Biol Soc 2007:2007:410-4.

2. Rossi S, Baruffi S, Bertuzzi A, et al. Ventricular activation is impaired in aged rat hearts. Am J Physiol-Heart C 2008;295:H2336-47. 